\title{
THE MASS DISTRIBUTION IN THE CENTRAL FEW PARSECS OF THE GALAXY
}

\author{
JOHN H. LACY \\ Astronomy Department \\ University of Texas \\ Austin, Texas, 78712 \\ U.S.A.
}

\begin{abstract}
The determination of the mass distribution in the central few parsecs of the Galaxy, primarily from the ionized gas dynamics, is discussed. The gas motions are described and interpreted assuming that the orbits are determined by gravitational forces. It is shown that there is good evidence for a central mass of $\sim 2 \times 10^{6} \mathrm{M}_{\odot}$. The primary uncertainty in this conclusion results from the possibility of significant magnetic forces. In the absence of corroborating evidence, the case for a massive black hole cannot be considered proven.
\end{abstract}

\section{Introduction}

Probably the most important questions we can ask about the center of the Galaxy is: is there a dominant central object and, if so, what is its nature? The most direct way of determining whether a very massive object lies at the center is by measuring the gravitational potential through the motions of gas and stars near the center.

In this review, I discuss the determinations of the gravitational potential and the uncertainties in those determinations. I concentrate on the gas motions, first summarizing what is seen, then describing the conclusions, and finally discussing the uncertainties. This review is complementary to those in this volume by Genzel, who discusses the neutral gas, Serabyn, who discusses the ionized gas, Sellgren, who discusses the stars, and Townes, who gives an overview of all phenomena in the central region. The reader is also referred to the review by Genzel and Townes (1987), which covers many of the topics discussed here.

In deriving masses and distances from the measurements, I use a distance to the Galactic Center of $8.5 \mathrm{kpc}$. If another value is preferred by the reader, dynamically derived masses and distances both scale as $R_{0}$.

\section{Gas Motions in the Inner Few Parsecs}

A sketch of the central few parsecs, from a point of view perpendicular to the plane of the circumnuclear disk, is shown in Figure 1. Lo and Claussen's (1983) $6 \mathrm{~cm}$ VLA map is shown in Figure 2 with several of the relevant features noted - see also Killeen and Lo (this volume).

Outside of $\sim 1.5 \mathrm{pc}$ the gas is predominantly molecular. The inner edge of the molecular disk, especially on the western side, is ionized, as is much of the gas inside $1.5 \mathrm{pc}$. Several ionized gas flows are seen which start at, or cross through, the disk and pass 


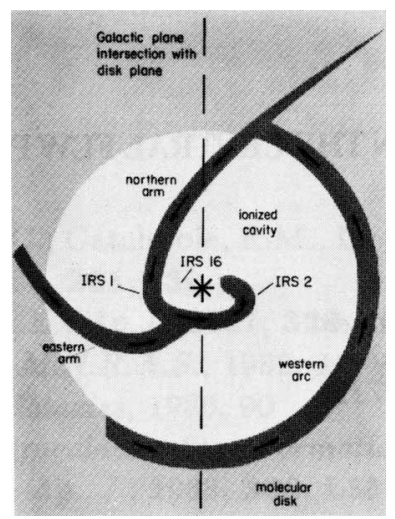

Figure 1: Sketch of the inner few parsecs of the Galaxy, viewed normal to the plane of the molecular disk and assuming that the sign of the inclination of all flows is the same.

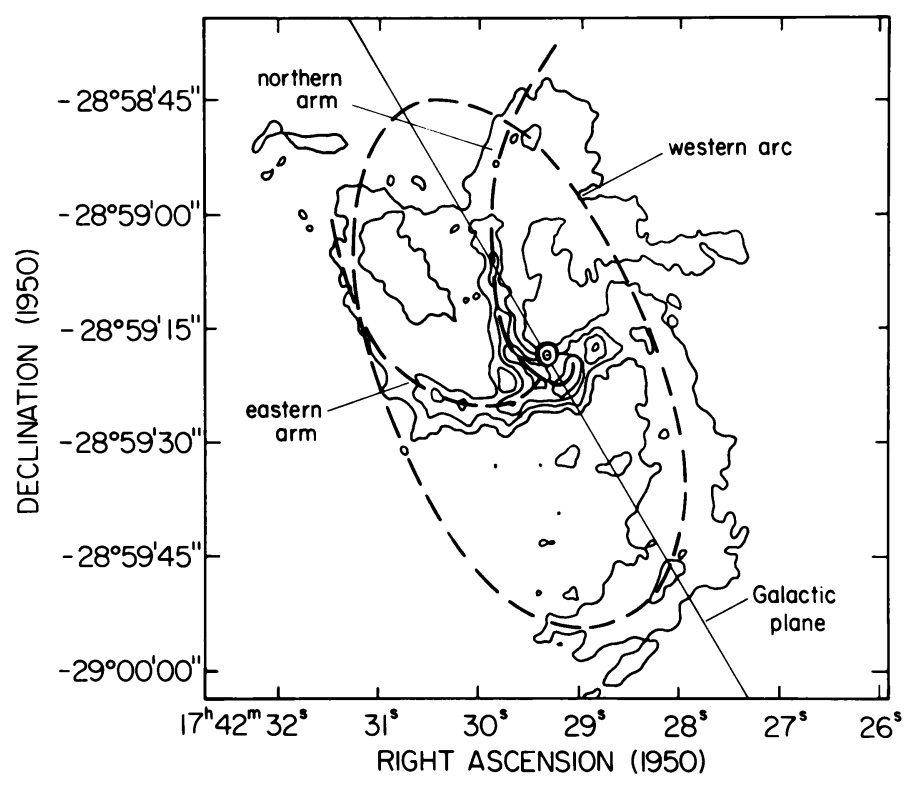

Figure 2: Paths of the various ionized gas flow superposed on the $6 \mathrm{~cm}$ VLA map of Lo and Claussen (1983). 
within $\sim 0.4 \mathrm{pc}$ of the center. Within $\sim 0.1 \mathrm{pc}$ of the center broad permitted lines are seen. The gas velocities are $\sim 110 \mathrm{~km} \mathrm{~s}^{-1}$ in the molecular disk, increasing to $\sim 400 \mathrm{~km} \mathrm{~s}^{-1}$ near the center. Our view of the Galactic Center is from an inclination of $\sim 70^{\circ}$ to the plane of the disk. The arrows showing the direction of motion in Figure 1 are shown assuming our view is from the right, but everything would appear the same if our view were from the left and the arrows were reversed. In fact, the sign of the inclination of various flows need not be the same. The obscuration of near-infrared sources by the western side of the disk (Gatley, Geballe, Genzel, this volume) indicates that the disk is seen from the right. If the inclinations of the ionized flows are the same as that of the disk, they are inflowing, but we do not yet have any direct evidence that this is the case.

The molecular gas, described in more detail by Genzel (this volume), forms a clumpy, flaring, and somewhat twisted disk with a $\sim 1.5 \mathrm{pc}$ radius inner hole. The rotational velocity in the disk is nearly constant at $\sim 110 \mathrm{~km} \mathrm{~s}^{-1}$ (corrected for inclination) from the inner edge out to $\sim 4 \mathrm{pc}$ (Güsten et al. 1987). It may fall off somewhat outside this, but the thickness of the disk makes the determination of the rotation curve more difficult toward the outside. Assuming that gravity is the dominant force on the gas and that the mass distribution is spherically symmetric, the mass interior to radius $r$ is given by: $M(r)=$ $\mathrm{v}^{2} \mathrm{r} / \mathrm{G}$. The mass inside $1.5 \mathrm{pc}$ is then $4.2 \times 10^{6} \mathrm{M} \odot$, and the mass increases linearly with radius out to $\sim 4 \mathrm{pc}$ where it is $11.2 \times 10^{6} \mathrm{M} \odot$. I won't quote an uncertainty on these numbers, since it is due more to possible errors in the assumptions made than in the measurements. Uncertainties, especially due to possible non-gravitational forces, are discussed in Section 4, below.

The various ionized gas flows are described by Serabyn (this volume). I simply quote his conclusions about the velocities and paths of the flows, and use these conclusions to infer the mass distribution. Again, non-gravitational forces are neglected for now. The ionized inner edge of the molecular disk, the "western arc", orbits at $\mathrm{r}=1.5 \mathrm{pc}$ with $\mathrm{v}=$ $110 \mathrm{~km} \mathrm{~s}^{-1}$, giving the same mass as inferred from the molecular gas. The streamer extending from the north end of the western arc and passing east of the center, the "northern arm", has been modeled by Serabyn and Lacy (1985). They conclude that it follows an eccentric orbit passing $\sim 0.4 \mathrm{pc}$ behind Sgr A* with about the same magnitude of inclination as the ring. Models of the orbit of the gas forming the northern arm require a mass inside $0.4 \mathrm{pc}$ of $>2.8 \times 10^{6} \mathrm{M} \odot$. The velocities in both the northern arm and the disk can be explained by a point-like mass of $\sim 2 \times 10^{6} \mathrm{M}_{\odot}$ in addition to a star cluster containing $\sim 2 \times 10^{6} \mathrm{M}_{\odot} \mathrm{pc}^{-1}$ (radius). Alternatively, the velocities can be explained by a mass distribution rising at least as steeply as $\rho(r) \propto r^{-2.7}$ inside $1.5 \mathrm{pc}$. It is not possible to explain both the northern arm and the western arc (or the disk) with a singular isothermal mass distribution of $\rho(r) \propto r^{2}$. Including a core radius would only increase the disagreement.

Other ionized flows, the "eastern arm" and the extension of the northern arm wrapping around south of $\mathrm{Sgr} \mathrm{A}^{*}$ (see Figures 1 and 2 ) have also been modeled and lead to essentially the same conclusion: $\mathrm{M}(\sim 0.25 \mathrm{pc}) \gtrsim 3 \times 10^{6} \mathrm{M}_{\odot}$, and the mass distribution cannot be that of an isothermal star cluster. Lacy (unpublished) has modeled the extended northern arm, and obtains an excellent fit to a large fraction of the observed ionized gas if the gas flows in the potential of $\mathrm{a} \sim 4 \times 10^{6} \mathrm{M}_{\odot}$ point mass, but is deflected to the west at IRS 1 (see Figure 2).

The highest ionized gas velocities, at least $\pm 400 \mathrm{~km} \mathrm{~s}^{-1}$, are seen near Sgr A* (Hall et al. 1982, Geballe et al. 1987). If these velocities were due to orbital motions, a non-stellar mass would almost certainly be required. However, the broad-line region has been spatially resolved (Geballe et al. 1987, confirmed by Lacy, Achtermann, and Bruce, unpublished) and does not exhibit ordered rotation. It seems more likely that the velocities are due to outflow, although several centers of outflow are needed. 


\section{The Mass Distribution}

The various measurements of enclosed mass within different radii are summarized in Figure 3, taken from Genzel and Townes (1987). Error bars have been deleted, but statistical errors are typically $\pm 50 \%$. The objects measured for each point are indicated on the figure; further details are given by Genzel and Townes. With the exception of the inner ionized gas flows, all measurements can be explained, within statistical and modeling uncertainties, by a $\rho \propto \mathrm{r}^{-1.8}$ mass distribution, as is suggested by the $2 \mu \mathrm{m}$ light distribution. Although the molecular gas motions suggest the presence of a central mass concentration, only the ionized gas flows come close enough to the center to allow a very strong statement. In addition, the mass inferred from stellar velocities (Sellgren, this volume) now has a statistical significance comparable to that inferred from the gas, and is free from some of the sytematic uncertainties in the interpretation of gas motions. Notably, the inferences from the stars are completely consistent with those from the gas.

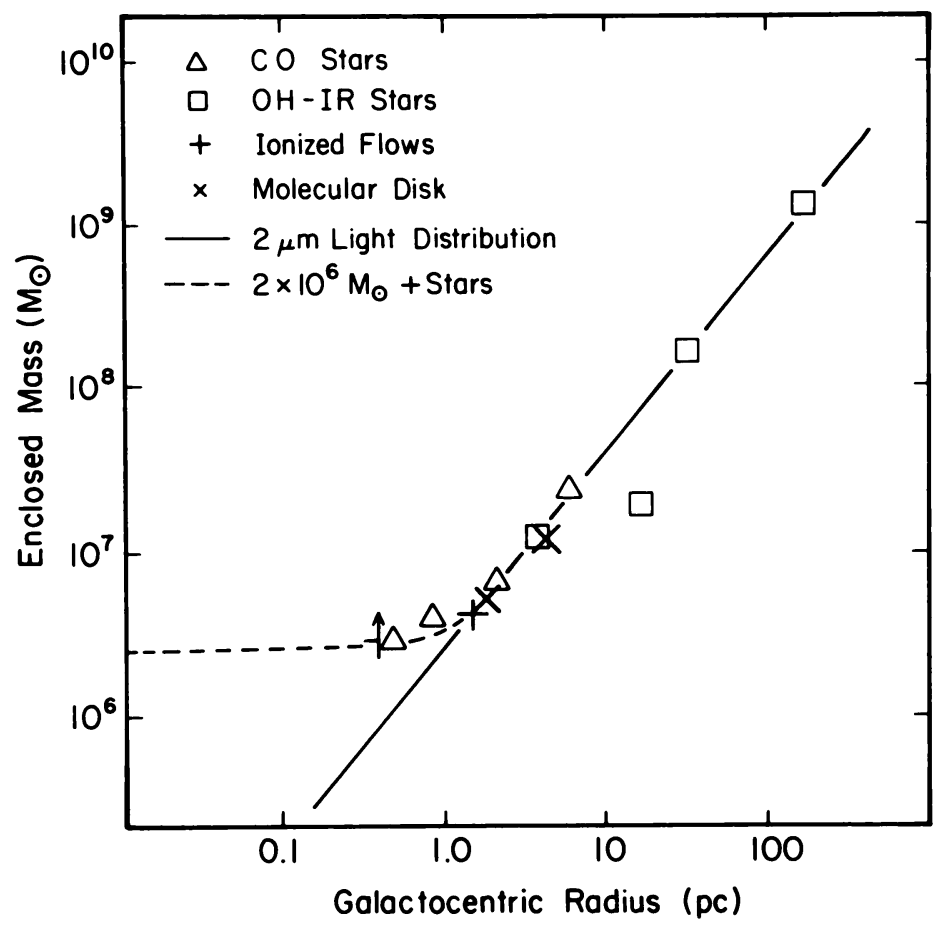

Figure 3: Enclosed mass versus Galactocentric radius. See Genzel and Townes (1987) for a description of the various measurements.

The best fit to all of the data consists of a central mass, presumably a black hole, of $\sim 2$ $3 \times 10^{6} \mathrm{M}_{\odot}$ embedded in a star cluster with $\mathrm{M} / \mathrm{L}_{2.2 \mu \mathrm{m}} \approx 1 \mathrm{M} \odot / \mathrm{L}_{\odot}$. Any core radius $\leqslant 1 \mathrm{pc}$ for the cluster is allowed. The derived mass distribution is then:

$$
M(r) \approx 2-3 \times 10^{6} M_{\odot}\left(1+r_{p c}^{1.2}\right)
$$


Note that $R_{0}=8.5 \mathrm{kpc}$ was assumed, and $\mathrm{M}$ and $\mathrm{r}$ scale as $R_{0}$.

\section{Uncertainties}

The discussion so far has assumed that the acceleration of the gas is caused solely by a centrally symmetric gravitational field. Disagreements about the validity of the derived mass distribution and particularly the inferred presence of a massive black hole most often center on the possibility of non-gravitational forces. Conclusions from the stellar velocities are not affected by this uncertainty, and so may ultimately supercede those from the gas.

Before discussing the difficulties in drawing conclusions from the gas motions, a significant advantage should be pointed out, however. Because the determination of the mass distribution from gas motions involves modeling of the velocity variations along extended flows, the initial conditions do not influence the conclusions and no assumption of virial equilibrium is made. In effect, the acceleration of the gas is determined from the rate of change of velocity with position. It should be noted that when a cloud is stretched into a streamer by tidal forces, the trailing part of the cloud follows essentially the same path as the leading part, so an observation of velocity and position at one time is equivalent to observing the path of a point mass along its orbit.

Turning to the difficulties in interpreting the gas motions, three possible nongravitational forces have been suggested: viscosity, radiation pressure, and magnetic fields. Quinn and Sussmann (1985) attempted to model both the western arc and the northern arm as one inward spiraling flow caused by drag due to a hot low density medium. Their predictions did not fit later observations, but could viscous forces be important? It is difficult to rule this possibility out, but if the ionized gas flows are infalling, as suggested above, a drag force would only increase the need for a central attractive force. On the other hand, if the gas is outflowing, a viscous force could mimic the effect of a central mass. Perhaps the best argument at present that viscosity is not important is that models without it fit the data better than those with it.

Radiation pressure could also have some effect. A gas clump or streamer subtending $0.1 \mathrm{sr}$ as seen from a $10^{7} \mathrm{~L}_{\odot}$ source would feel the same force as $0.1 \mathrm{M}_{\odot}$ the same distance from $3 \times 10^{6} \mathrm{M}_{\odot}$. This force would be negligible in the molecular ring, but possibly relevant for the ionized gas. Again, being a repulsive force, if it is present a larger mass concentration would be required .

The non-gravitational force most likely to seriously affect the gas motions is that due to magnetic fields. Aitken et al. (1986) observe polarized $10 \mu \mathrm{m}$ emission from dust in the ionized streamers, whereas Werner et al. (1988) observe polarized $100 \mu \mathrm{m}$ emission from dust in the molecular disk. The required field in the ionized region is in all positions aligned with the flows as modeled by Serabyn et al. (1988), while the field in the disk is in the plane of the disk. In both cases a field of $\gtrsim 10 \mathrm{mG}$ is required to align the emitting grains if the Davis-Greenstein alignment mechanism applies.

Two explanations for the alignment of the fields with the flows seem plausible: 1) the energy density in the field dominates over that in the flows and so the gas is constrained to move along the field lines, or 2) the field is relatively unimportant dynamically and is aligned by tidal stretching of the gas which carries the field along with it. In the first case, the filamentary structure of the ionized gas and the geometry of the flows would result from the field structure, and the velocities may have no direct relationship to the gravitational potential. In the second case, the filamentary structure would result from tidal stretching of the gas, and the strength of the field would be determined by a balance between tidal forces and gas and magnetic pressure forces. It should, in principle, be possible to choose between these options by comparing the magnitudes of the different 
forces acting on the gas or, equivalently, the energy densities. The energy densities associated with gas pressure, magnetic fields, gravitational potential, and tidal forces are:

$$
\begin{aligned}
& \mathrm{E}_{\mathrm{p}} \approx \mathrm{nkT} \quad \approx 10^{-7} \mathrm{erg} \mathrm{cm}^{-3} \quad \text { for } \mathrm{n}=10^{5} \mathrm{~cm}^{-3}, \mathrm{~T}=7000 \\
& \mathrm{E}_{\mathrm{m}} \approx \frac{1}{8 \pi} \mathrm{B}^{2} \approx 4 \times 10^{-6} \mathrm{erg} \mathrm{cm}^{-3} \text { for } \mathrm{B}=10 \mathrm{mG} \\
& \mathrm{E}_{\mathrm{g}} \approx \frac{\mathrm{GM} \rho}{\mathrm{r}} \approx 3 \times 10^{-5} \mathrm{erg} \mathrm{cm}^{-3} \text { for } \mathrm{M}=3 \times 10^{6} \mathrm{M}_{\odot}, \mathrm{r}=1 \mathrm{pc} \\
& \mathrm{E}_{\mathrm{t}} \approx \frac{\mathrm{GM} \rho}{\mathrm{r}^{3}}(\Delta \mathrm{r})^{2} \approx 3 \times 10^{-7} \mathrm{erg} \mathrm{cm}^{-3} \text { for } \Delta \mathrm{r}=0.1 \mathrm{pc}
\end{aligned}
$$

where the gravitational field is that of a point mass and the tidal energy refers to the work required to stretch or compress a cloud against the tidal force. With the numbers assumed, $\mathrm{n}=10^{5} \mathrm{~cm}^{-3}$ and $\mathrm{B}=10 \mathrm{mG}$, gravity dominates the motions, but the tidal force is insufficient to form a cloud into a streamer. Unfortunately, our knowledge of the magnetic fields and gas density are so uncertain (by at least an order of magnitude) that magnetic fields could totally dominate the motion or be totally negligible.

This discussion leaves us very uncertain as to whether or not the derivation of a gravitational potential from the gas motions is valid. This situation is not likely to change until better determinations of the magnetic field or magnetohydrodynamic models of the gas motions are made. However, we do know that the gravitational potential derived from stellar velocities is entirely consistent with that derived from the gas. Could this occur if the gas motions were channeled by magnetic fields? The answer may be yes if the gas is pulled along fixed field lines by gravity. In this case the flow geometries derived by Serabyn $e t$ al. would be essentially valid, and approximately the same gravitational force would be required. Why the field geometry so closely matches the geometry of free-fall orbits would be a mystery, however.

I now turn briefly to the uncertainties in the derivation of the gravitational potential from stellar velocities. Until recently, the major uncertainty was due to inadequate statistics. The situation has improved significantly with the work of Rieke and Rieke (1988) and McGinn et al. (1988), however. At present, the two primary uncertainties are probably the lack of knowledge of the spatial distribution of the stars which goes into the equation of stellar hydrodynamics, and the possibility of anisotropic motions of the stars. The first of these can be resolved with sufficient effort. The second will be much more difficult. For now, I will just note that the conclusions from the stars and the conclusions from the gas are in complete agreement, suggesting that the difficulties in both approaches may be less severe than they seem.

\section{Is There a Black Hole?}

Qualitatively, the answer to the central question of the existence of a massive black hole has not changed for the last ten years. The dynamical evidence has always indicated that the answer is yes, with decreasing but still substantial uncertainties. On the other hand, other forms of evidence have always been very difficult to find. Sgr A* is clearly an unusual object (see Lo, this volume), but seems not to require a $10^{6} \mathrm{M}_{\odot}$ black hole, whereas the positron annihilation radiation (see Lingenfelter and Ramaty, this volume) has not been conclusively associated with the very center. The papers by Phinney and Ozernoy (this volume) discuss many of the observable consequences of a massive black hole, and neither 
concludes that one has been seen. I would like to mention two consequences discussed by them and others which seem to me to be particularly relevant.

If a black hole is present at the Galactic Center, one might expect to see the radiation emitted by the accreting gas. However, no one source stands out at any infrared wavelength. In particular, there is very little infrared emission from the direction of Sgr $A^{*}$. It has been shown that the ionizing ultraviolet radiation and a substantial fraction of the luminosity could come from a single source which would not be prominent in the infrared if its spectrum were like that of an O9 $(35,000 \mathrm{~K})$ star (Serabyn and Lacy 1985 , Geballe et al. 1984). Standard ( $\alpha$ disk) models of black hole accretion can produce the required ultraviolet radiation, but do not predict spectra falling as fast as $\mathrm{O}$ star spectra in the infrared. Lacy et al. (1982) show that such a disk should be an order of magnitude brighter than any observed source. Unless an accretion disk model which more nearly matches an $\mathrm{O}$ star can be made, it appears that the black hole does not provide the ionizing radiation.

Most calculations also predict a cusp of stars should be found around a black hole (see Phinney, this volume). The observational results on this question are rather ambiguous. Allen et al. (1983) find evidence for a cusp, whereas Rieke and Lebofsky (1987) argue that the distribution of faint stars is nearly constant within $\sim 1 \mathrm{pc}$. In any case, there is no cusp centered on Sgr A*, as pointed out by Allen and Sanders (1986). More theoretical and observational work is needed in this area.

In conclusion, the dynamical evidence continues to support the suggestion of a massive black hole at the Galactic Center, but corroborating evidence is sadly lacking. We badly need an unambiguous and testable prediction which can answer this important question.

This work was supported by the NSF and the State of Texas.

\section{References}

Aitken, D. K., Roche, P. F., Bailey, J. A., Briggs, G. P., Hough, J. H., \& Thomas, J. A. (1986). M.N.R.A.S. 218: 363.

Allen, D. A., Hyland, A. R., \& Jones, T. J. (1983). M.N.R.A.S. 204: 1145.

Allen, D. A. \& Sanders, R. M. (1986). Nature 319: 191.

Geballe, T. R., Krisciunas, K. L., Lee, T. J., Gatley, I. \& Wade, R., et al. (1984). Ap. J. 284: 118.

Geballe, T. R., Wade, R., Krisciunas, K. L., Gatley, I. \& Bird, M. C. (1987). Ap. J. 320: 562 .

Genzel, R. \& Townes, C. H. (1987). Ann. Rev. Astr. Ap. 25: 377.

Güsten, R., Genzel, R., Wright, M. C. H., Jaffe, D. T., Stutzki, J. \& Harris, A. I. (1987). Ap. J. 318: 124.

Hall, D. N. B., Kleinmann, S. G. \& Scoville, N. Z. (1982). Ap. J. Lett. 262: L53.

Lacy, J. H., Townes, C. H. \& Hollenbach, D. J. (1982). Ap. J. 262: 120.

Lo, K. Y. \& Claussen, M. J. (1983). Nature 306: 647.

McGinn, M. T., Sellgren, K., Becklin, E. E., Hall, D. N. B. \& Gatley, I. (1988). Ap. $J .$, in press.

Quinn, P. J. \& Sussman, G. J. (1985). Ap. J. 288: 377.

Rieke, G. H. \& Lebofsky, M. J. (1987). The Galactic Center, ed. D. C. Backer, p. 91.

Rieke, G. H. \& Rieke, M. J. (1988). Ap. J. Letts. 330: L33.

Serabyn, E. \& Lacy, J. H. (1985). Ap. J. 293: 445.

Serabyn, E., Lacy, J. H., Townes, C. H. \& Bharat, R. (1988). Ap. J. 326: 171.

Werner, M. W., Davidson, J. A., Morris, M., Novak, G., Platt, S. R. \& Hildebrand, R. H. (1988). Ap.J. in press. 